\title{
COMPRENSIÓN LECTORA Y RECONOCIMIENTO DE PALABRAS
}

\section{READING COMPREHENSION AND RECOGNITION WORDS}

\author{
Alejandra Balbi \\ Ariel Cuadro \\ Daniel Trías \\ Universidad Católica del Uruguay, Uruguay
}

\begin{abstract}
Resumen: Desde el ya clásico modelo simple de lectura propuesto por Gough y Tunmer (1986), numerosos estudios han pretendido demostrar la contribución relativa e independiente de la decodificación a la comprensión. El presente estudio tiene por objetivo precisar esta relativa independencia, al correlacionar habilidades, ya no de decodificación, sino de procesamiento léxico-semántico y fluidez, con la comprensión de textos, en sujetos de $4^{\circ}, 5^{\circ}$ y $6^{\circ}$ grado escolar. Los resultados evidencian a nivel general $\mathrm{y}$ en los tres grados escolares, una correlación positiva y moderada entre las variables estudiadas. Se discuten estos resultados y sus implicaciones educativas.
\end{abstract}

Palabras clave: decodificación, comprensión, dificultades.

\begin{abstract}
Since the classic simple reading model proposed by Gough and Tunmer (1986), numerous studies have intended to demonstrate the relative and independent contribution of the decodification to the comprehension. The objective of the present study is to specify these relative independence by correlating skills, not of decodification, but of the "lexic-semantic" processing and fluency, with the reading comprehension in 4th, 5th and 6th grade individuals. The results show, in the hole sample and in each of the three school grades, a positive and moderate correlation between the variables studied. These results and its educative implications are discussed.
\end{abstract}

Keywords: decodification, reading comprehension, difficulties.

\section{INTRODUCCIÓN}

A La comprensión de textos es un proceso complejo que involucra la participación de diferentes componentes cognitivos y socioeducativos (Kintsch y Rawson, 2007) Esta interdependencia lo hace un proceso dinámico y muy sensible a la variación individual por lo que su estudio plantea limitaciones metodológicas (Nation, 2007; Kelso, Fletcher y Lee, 2007) que explican que los estudios de la lectura se hayan orientado a los procesos de reconocimiento de palabras o decodificación (Johnston, Barnes y Desrochers, 2008, Perfetti, 2008). Aunque resulta indiscutible la importancia de los mismos en la comprensión, no es suficiente para explicar todos procesos involucrados en la comprensión de textos (Perfetti, 2007).
Desde el ya clásico modelo simple de la lectura propuesto por Gough y Tunmer (1986) donde propone la comprensión de textos como el resultado de dos componentes: lenguaje y decodificación, numerosos estudios se han sucedido para ratificar y refutar este argumento. Bertelson (1986) afirma que una vez que el problema de la identificación de palabras escritas ha sido resuelto no queda (casi) nada por hacer para ser un buen lector. Estudios posteriores (Gough, Juel y Grittith, 1992; Hoover y Gough, 1990) analizan la variación de la correlación entre decodificación (D), lenguaje (L) y comprensión de textos (CT) entre los primeros años de escolaridad y el inicio de la secundaria, encontrando que mientras al inicio la decodificación es el más importante $(r(C T x D)=.61$ y $r(C T x L)=.39)$, estos valores

Correspondencia: Alejandra Balbi. Facultad de Psicología. Universidad Católica del Uruguay. Correo electrónico: abalbi@ucu. edu.uy. 
se invierten en etapa liceal $(r(C T x L)=.68$ y $r(C T x D)=.39)$. La lógica de este punto de vista sostiene que el déficit de la comprensión lectora no puede ser específico debe estar relacionado con deficiencias en uno o ambos de sus componentes.

Si bien parece evidente que la ineficiencia de decodificación llevará a una comprensión lectora con dificultades (Perfetti, 1985), numerosos estudios indican que no todos los niños que tienen dificultades de comprensión tienen deficiencias en decodificación. Como indica Perfetti (2005) la identificación de palabras parece no explicar toda la historia en comprensión. La evidencia parece clara en identificar grupos de niños (Georgiou, GK., Das, JP. Y Hayward, D., 2009, Kelso et al., 2007 Cain \& Oakhill, 1999; Nation y Snowling, 1998; Stothard y Hulme, 1992) y adultos (Hart, 2005; Landi, 2005), cuyos problemas con la comprensión no están asociados con la decodificación de la palabra.

Oakhill, Kain y Bryant (2003) sugieren que decodificación y comprensión hacen contribuciones independientes a la lectura global, esto implicaría no sólo que subyacen capacidades diferentes a cada una, sino que el desarrollo de cada una de estas capacidades, debiera ser independiente también. Estos autores, en un estudio longitudinal con niños de 7 y 8 años $\left(1^{\circ}\right) 8$ y 9 años $\left(2^{\circ}\right)$, muestran disociación entre lectura de palabras y comprensión. La diferencia en comprensión de textos se explica por las variables de los procesos de integración de significados, monitoreo y memoria de trabajo y no por la varianza en lectura de palabras.

Pazaglia, Cornoldi y Tressoldi (1993) muestran en un estudio de revisión que decodificación y comprensión lectora aparecen al menos parcialmente separadas, relacionadas con diferentes habilidades cognitivas subyacentes, y con diferente sensibilidad a los programas de intervención. En un estudio posterior Cornoldi, De Beni y Pazzaglia (1996) examinaron los perfiles cognitivos de los malos comprendedores encontrando debilidades en los procesos de integración, monitoreo y memoria de trabajo, aunque no en la misma medida para todos los niños. Cornoldi (1996) propone una actitud de cautela al interpretar la relación entre estos procesos y la comprensión de textos, ya que la variación de perfiles encontrada indica que la relación no tiene por qué ser causal ni permanente. Otros estudios longitudinales (Muter,
Hulme, Snowling, 2004) sugieren que los predictores del desarrollo de la lectura de palabras son muy diferentes de los de la comprensión.

Según los hallazgos de Johnston et al. (2008), las inferencias puente entre dos ideas adyacentes en un texto, son hechas más frecuentemente por los niños mayores que por los más pequeños, en tanto las inferencias globales que implican una conexión entre frases alejadas en el texto, son tan complejas para los sujetos de menor edad como para los mayores. Las dificultades para decidir cuándo realizar la inferencia y usar el contexto parecen ser en la mayoría de los estudios la fuente de explicación de las diferencias individuales en comprensión. Estudios en adultos con pobre comprensión muestran que estos activan una amplia gama de significados de las palabras, pero tienen dificultad para suprimir los significados irrelevantes de acuerdo a las limitaciones que impone el contexto de referencia.

Storch y Whitehurst (2002) estudian niños desde preescolar hasta cuarto escolar, identificando que las habilidades relacionadas con la decodificación predicen fuertemente el nivel lector durante los primeros años escolares, pero pierden posición en cuarto año. En este grado escolar las habilidades de precisión y comprensión lectora se diferencian mostrando contribuciones independientes.

Nation y Snowling $\left(1998^{a}\right)$ identifican un grupo de pobres comprendedores que leen palabras y pseudopalabras en los niveles adecuados, y sus habilidades fonológicas se muestran bien desarrolladas. Concluye que es poco probable que esos niños dediquen excesivos recursos a la identificación de la palabra y decodificación, o que su comprensión lectora esté muy comprometida por la ineficacia de los procesos de identificación de palabras. Sin embargo, los resultados muestran que otros aspectos del procesamiento léxico son débiles en los pobres comprendedores relacionados con la hipótesis léxico-semántica propuesta por Perfetti (2008) quien sugiere que la relación no estaría dada con la decodificación sino con la activación del significado pertinente en la oración. Propone que buenos y malos comprendedores se diferencian por la flexibilidad de sus representaciones semánticas de las palabras, una dimensión de la calidad del léxico (Perfetti y Hart, 2001), y que esta variabilidad tiene consecuencias para la recuperación de los significados de la palabra así como de la selección de los mismos 
en el contexto apropiado. Este fracaso puede suponer deficiencias en la integración de procesamiento de textos, en la capacidad de vincular significados de las palabras en el contexto de una frase adecuada, con independencia de la habilidad en decodificación.

Con esta evidencia, en el presente trabajo mostramos los resultados de un estudio en el que se analiza la relación entre decodificación léxico-semántica y comprensión de textos en una muestra de escolares de Montevideo.

Dado que existe suficiente evidencia para demostrar que decodificación y comprensión de textos siguen cursos de desarrollo independientes, proponemos una tarea que involucra decodificación en un contexto frase. La formulación de las preguntas que guían nuestro estudio sería: ¿los procesos de decodificación en un contexto de frase con significado, están relacionados con los procesos de comprensión de textos más complejos?; ¿la relación entre los procesos a estudiar, se mantiene estable en los diferentes grados escolares?; ¿es posible discriminar las habilidades necesarias para comprender el texto-base, de aquellas necesarias para comprender el modelo de situación?

Se establecen perfiles lectores específicos de acuerdo a sus diferentes componentes y se interpretan en el marco de los estudios de la contribución de la decodificación a la comprensión lectora.

\section{MÉTODO}

\section{Participantes}

La muestra estuvo compuesta por 302 estudiantes de los cuales 105 son de 4to año escolar, 105 de $5^{\circ}$ y 92 de $6^{\circ}$ grado escolar. Corresponden a dos escuelas urbanas y privadas de Montevideo de procedencia socio-cultural medio-alta.

\section{Instrumentos de evaluación}

a) Comprensión de Textos: esta prueba ha sido desarrollada específicamente para este trabajo y consiste en evaluar el nivel de comprensión lectora en modalidad silenciosa con preguntas que demandan niveles de comprensión superficial y profunda, así como tareas de titular el texto y explicar a través de la escritura el tema de los mismos. Los textos empleados fueron "Los esquimales" y "Los papúes australianos"- de la Batería PROLEC-SE (Cuetos y Ramos, 1997), con las siguientes modificaciones. Se suprime el título de los textos, el texto está presente y disponible para el sujeto, se eliminan las preguntas literales y se propone la respuesta de 20 preguntas con modalidad múltiple (cuatro) opciones. Estas preguntas demandan tareas de comprensión del texto base, a nivel local y a nivel global, así como demandas de elaboración del modelo de situación. Sólo una respuesta es la correcta, el acierto contabiliza con un punto por lo que se puede alcanzar un máximo de 20. Se llamará a esta medida Comprensión. La fiabilidad de la prueba analizada a través del alfa de Cronbach es de .73 .

Por otra parte se agregan dos nuevos ítems a cada texto que responden a la construcción de procesos más próximos a los procesos de integración o construcción del modelo de situación: titular el texto y explicar en no más de tres proposiciones el tema sobre el que trata. Esta segunda prueba además de demandar tareas de comprensión profunda, se diferencia de la primera medida de comprensión por involucrar escritura. Estos cuatro ítems se pueden puntuar con 2, 1 o 0 por lo que se puede alcanzar un máximo de 8 puntos. Se llamará a esta medida Comprensión 1. Esta prueba tiene una confiabilidad de 0.5 según el alfa de Cronbach.

De este modo se obtienen dos medidas de comprensión: 20 ítems que indagan procesos del texto base, aunque no literales; y 4 ítems que indagan básicamente modelo de situación (ver Tabla 1). Las medidas de Comprensión fueron sometidas a un análisis de dificultad del ítem, conservando únicamente aquellos ítems con buen y muy buen poder de discriminación.

Prueba de lectura TECLE: para medir la capacidad de reconocimiento de palabras se ha utilizado Test de Eficacia Lectora (TECLE) de J. Marín y M, Carrillo (Cuadro et al., 2009). Esta compuesta de un total de 64 ítems que contienen una frase encabezado y cuatro opciones de respuesta. A la frase encabezado le falta la última palabra, que debe ser seleccionada entre las cuatro opciones de respuestas. Las tres opciones falsas son dos pseudopalabras que constituyen pares mínimos ortográficos y fonológicos de la respuesta correcta y por último, 
una palabra que resulta inadecuada desde el punto de vista semántico y/o sintáctico para completar la frase. La prueba esta cronometrada, por lo que la selección de alternativas debe producirse con el menor consumo de tiempo disponible. La puntuación de esta prueba es el número de respuestas correctas emitidas.
Con las autoridades educativas como mediador, se solicitó la aprobación de las familias de los alumnos, se les informó sobre las características del estudio, la confidencialidad de los datos y que dichas autoridades educativas serían el nexo para que las familias pudieran acceder a la información de cada alumno.

Tabla 1. Ejemplo de ítems de las medidas de comprensión

\begin{tabular}{ll}
\hline Prueba Comprensión & Prueba Comprensión 1 \\
\hline Ejemplo de preguntas & Ejemplo de ítem Título \\
\hline $\begin{array}{ll}\text { ¿Cuándo pueden navegar entre los bloques de } \\
\text { hielo? }\end{array}$ & $\begin{array}{l}\text { ¿Qué título te parece más apropiado para el } \\
\text { texto? }\end{array}$ \\
\hline $\begin{array}{l}\text { 1. Cuando el deshielo permite la formación de } \\
\text { canales }\end{array}$ & 1. El problema de la falta de luz solar \\
\hline 2. De noviembre a enero & 2. Los beneficios de la fauna para los esquimales \\
\hline 3. Antes que se desprendan los icebergs & 3. La naturaleza \\
\hline 4. Cuando los ríos se tornan menos peligrosos & 4. Un pueblo que lucha por su supervivencia \\
\hline & Ejemplo de ítem \\
\hline & Explica brevemente de qué se trata el texto \\
\hline Puntajes: 100 & Puntaje: 2,100 \\
Máximo: 20 & Máximo: 8 \\
\hline
\end{tabular}

\section{Procedimiento}

La aplicación de los instrumentos de evaluación ocupó en cada colegio una sesión colectiva de 50 a 60 minutos aproximadamente. En el caso de los grupos de $4^{\circ}$ año la evaluación se realizó en dos sesiones: TECLE y Texto Esquimales en la primera; Texto Papúes en la segunda, en un período no mayor de una semana.

El orden de aplicación consistió en comenzar por la Prueba TECLE, dado que se debe cronometrar su inicio y final. A continuación se entrega el primer texto (Los Esquimales) y se realiza la lectura conjunta de la consigna, dónde se enfatiza a los alumnos que pueden tomar el tiempo que necesiten y consultar el texto cuántas veces requieran. Se indica que cuando terminen el primero, seguirán con un segundo y último texto que deben realizar del mismo modo. Los administradores fueron entrenados sobre cómo estar accesibles a las consultas de los alumnos, sin agregar información adicional alguna a la aportada durante la consigna.
De forma directa se mantuvo -durante todo el proceso- una comunicación abierta entre las familias y los responsables de la investigación.

\section{RESULTADOS}

Los resultados obtenidos en las variables estudiadas de: decodificación, comprensión de texto base (Comprensión) y comprensión de procesos globales (Comprensión 1) constituyen el conjunto de datos que analizaremos.

En la Tabla 2 se muestran la medias y las desviaciones típicas de las puntuaciones directas de las distintas tareas aplicadas en función del grado escolar.

En la Tabla 3 se presentan los resultados de las correlaciones entre la prueba TECLE con puntuaciones directas y las dos medidas de comprensión de textos con toda la muestra y por curso.

Tabla 2. Media y desviación típica de las puntuaciones directas de las tareas aplicadas en cada grado escolar

\begin{tabular}{lllll}
\hline Grupo & N & TECLE & Comprensión & Comprensión 1 \\
\hline & & Media (DE) & Media (DE) & Media (DE) \\
$\mathbf{4}^{\circ}$ & 105 & $30.71(8.68)$ & $10.66(3.14)$ & $4.2(1.71)$ \\
$\mathbf{5}^{0}$ & 105 & $35.03(9.47)$ & $9.58(3.57)$ & $4.16(1.71)$ \\
\hline $\mathbf{6}^{0}$ & 92 & $39.58(9.74)$ & $13.94(3.06)$ & $4.52(1.74)$ \\
\hline
\end{tabular}


Tabla 3: Tabla resumen del coeficiente de Pearson entre los resultados del

TECLE y las dos medidas de comprensión de textos. ${ }^{* *} \mathrm{p}<.001 * \mathrm{p}<0.05$

\begin{tabular}{|c|c|c|c|c|c|}
\hline \multirow[t]{2}{*}{ Grado } & \multirow[t]{2}{*}{$N$} & \multirow{2}{*}{$\begin{array}{c}\text { TECLE/ } \\
\text { Comprensión }\end{array}$} & \multirow[t]{2}{*}{ TECLE/ } & \multicolumn{2}{|c|}{ Comprensión/ } \\
\hline & & & & Comprensión 1 & Comprensión 1 \\
\hline 4 & 105 & $0.386^{* *}$ & $0.214^{*}$ & & $0.441^{* *}$ \\
\hline 5 & 105 & $0.378^{* *}$ & $0.337^{* *}$ & 0.470 ** & \\
\hline 6 & 92 & $0.295^{\star *}$ & $0.339^{* *}$ & $0.450^{\star *}$ & \\
\hline total & 302 & $0.401^{* *}$ & $0.303^{\text {** }}$ & $0.452^{\star *}$ & \\
\hline
\end{tabular}

El primer análisis informa de una correlación moderada entre los diferentes componentes de la lectura evaluados ( $r=.40, r=.30$ y $r=.45)$. Se realiza un segundo análisis donde se establecen cuatro niveles de desempeño por grado escolar, para las dos variables de lectura que muestran mayor fiabilidad: Tecle y Comprensión. Se utilizaron los centiles de la Prueba TECLE y se establecieron cuartiles para las pruebas de Comprensión en función de la media y sus desvíos estándar. Diferenciamos así cuatro niveles de desempeño que se presentan en la Tabla 4. Con la prueba $X^{2}$ se buscó analizar la relación entre los niveles identificados en la Prueba
TECLE y los niveles de comprensión, ambos agrupados en cuartiles (ver Tabla 5).

Encontramos que las variables están asociadas de forma significativa en $4^{\circ}$ y $5^{\circ}$ grado escolar, no así en $6^{\circ}$. Se calcula la fuerza de la relación a través del coeficiente $\mathrm{V}$ de Cramer que es el procedimiento indicado cuando las tablas tienen más de dos categorías por variable, y se evidencia una fuerza de asociación de moderada a baja.

Se presentan a continuación las tablas 6,7 y 8 de contingencia por grado escolar, que se utilizarán para la discusión de la relación entre los niveles.

Tabla 4. Categorías descriptivas de los niveles lectores

\begin{tabular}{|c|c|}
\hline Grupo 1 & $\begin{array}{l}\text { Malos decodificadores } \\
\text { Malos comprendedores }\end{array}$ \\
\hline Grupo 2 & $\begin{array}{l}\text { Promedio-Bajo Decodificadores } \\
\text { Promedio-Bajo Comprendedores }\end{array}$ \\
\hline Grupo 3 & $\begin{array}{l}\text { Promedio-Alto Decodificadores } \\
\text { Promedio-Bajo Comprendedores }\end{array}$ \\
\hline Grupo 4 & $\begin{array}{l}\text { Excelentes-Decodificadores } \\
\text { Excelentes-Comprendedores }\end{array}$ \\
\hline
\end{tabular}

Tabla 5. Grado de la relación entre las variables

\begin{tabular}{|c|c|c|c|c|}
\hline Grado & $\mathbf{N}$ & $\boldsymbol{\chi}^{\mathbf{2}}$ & $\mathbf{p}$ & $\mathbf{V}$ de Cramer \\
\hline 4 & 105 & 17,47 & 0,042 & 0.236 \\
\hline 5 & 105 & 18.012 & 0.035 & 0.231 \\
\hline 6 & 92 & 14,474 & 0.097 & 0.239 \\
\hline
\end{tabular}

Tabla 6: Tabla de contingencia entre los cuartiles de TECLE y los niveles de comprensión de textos en $4^{\circ}$ grado escolar

\begin{tabular}{|l|lllll|}
\hline Niveles & \multicolumn{5}{|l}{ Niveles de Comprensión } \\
TECLE
\end{tabular}

Tabla 7: Tabla de contingencia entre los cuartiles de TECLE y los niveles de comprensión de textos en $5^{\circ}$ grado escolar.

\begin{tabular}{|c|c|c|c|c|c|}
\hline Niveles & \multicolumn{4}{|c|}{ Niveles de Comprensión } & Total \\
\hline & $\mathrm{MC}$ & $\mathrm{BC}$ & $\mathrm{AC}$ & EC & \\
\hline MD & $4(12,5 \&)$ & $19(59.4 \%)$ & $7(21.9 \%)$ & $2(6,3 \%)$ & 32 \\
\hline BD & $4(15.4 \%)$ & $12(46.2 \%)$ & $8(30.8 \%)$ & $2(7.7 \%)$ & 26 \\
\hline D & $1(4.3 \%)$ & $10(43.5 \%)$ & $10(43.5 \%)$ & $2(8.7 \%)$ & 23 \\
\hline ED & $0(0 \%)$ & $8(33,3 \%)$ & $8(33,3 \%)$ & $8(33,3 \%)$ & 24 \\
\hline Total & $14(13.3 \%)$ & $40(38.1 \%)$ & $32(30,5 \%)$ & $14(13,3 \%)$ & 105 \\
\hline
\end{tabular}


Tabla 8: Tabla de contingencia entre los cuartiles de TECLE y

los niveles de comprensión de textos en $6^{\circ}$ grado escolar.

\begin{tabular}{|c|c|c|c|c|c|}
\hline Niveles & Niveles de C & omprensión & & & Total \\
\hline & $\mathrm{MC}$ & $\mathrm{BC}$ & $A C$ & EC & \\
\hline MD & $9(37.5 \%)$ & $8(33.3 \%)$ & $6(25 \%)$ & $1(4.2 \%)$ & 24 \\
\hline BD & $3(14.3 \%)$ & $4(19 \%)$ & $11(52.4 \%)$ & $3(14.3 \%)$ & 21 \\
\hline AD & $1(6 \%)$ & $7(41 \%)$ & $6(35 \%)$ & $3(18 \%)$ & 17 \\
\hline ED & $4(13 \%)$ & $9(30 \%)$ & $11(37 \%)$ & $6(20 \%)$ & 30 \\
\hline Total & $17(18.5 \%)$ & $28(30.4 \%)$ & $34(37 \%)$ & $13(14 \%)$ & 92 \\
\hline
\end{tabular}

\section{DISCUSIÓN Y CONCLUSIONES}

Se evaluaron diferentes componentes de lectura: léxico-semánticos (TECLE) y comprensión lectora en alumnos de Cuarto, Quinto y Sexto grado escolar. Los resultados obtenidos evidencian una progresión regular a medida que avanza la escolaridad, en los procesos léxicosemánticos, mientras que la comprensión de textos no se diferencia entre Cuarto y Quinto.

Aspectos metodológicos deben ser considerados ya que Cuarto año fue el único grupo donde la aplicación se realizó en dos sesiones. El hecho que más tiempo posibilitara a los alumnos de Cuarto alcanzar los mismos resultados que los alumnos del curso superior, en tareas de alta complejidad cognitiva, remite a un análisis teórico respecto a la naturaleza de los procesos de comprensión lectora, a diferencia de los procesos léxico-semánticos. Cain (2007) encuentra que diferentes grupos lectores se benefician de estrategias contextuales para inferir el significado de palabras nuevas, por el sólo hecho de intentarlo, con independencia de los programas de intervención. Según Perfetti (1994, p. 885), "hay espacio para un montón de cosas que van mal, cuando falla la comprensión", por lo que un buen resultado también puede ser obtenido a partir de otros recursos cognitivos interdependientes que actúan en paralelo y retroalimentan la comprensión. Es probable entonces, que los lectores más jóvenes de nuestro estudio, con más tiempo y oportunidades para abordar la comprensión del texto, hayan encontrado estrategias eficaces para aventajar en rendimiento a los lectores de Quinto año.

Los análisis de correlación general $(r=0.401$. $p<0.01)$ se mantienen en sintonía con los ha llazgos teóricos al evidenciar que las variables estudiadas están asociadas, aunque de forma moderada. Una correlación positiva pero no perfecta (Nation, 2007) admite aceptar que algunos sujetos realicen adecuadamente uno de los componentes y no el otro, es decir, que ambos componentes puedan ser claramente disociados (Gough et al. 1996). Nuestra expectativa inicial era encontrar una asociación más fuerte entre las variables al proponer una tarea que implica precisión en la lectura pero en un contexto de significado-frase, a diferencia de otros estudios de correlación que ponen en juego lectura de palabras aisladas o pseudopalabras (Nation y Snowling, 1998 a). Este hallazgo confirma el supuesto de que ambos componentes de lectura, aunque asociados, son sostenidos por habilidades independientes en el curso de su desarrollo (Oakill, 2003). Mientras que decodificación se encuentra altamente sostenida por habilidades fonológicas y de velocidad de nominación (Cuadro, 2007) comprensión de textos se relaciona con habilidades lingüísticas ( $\mathrm{Na}$ tion, 2007) y procesos de alto nivel : inferencias, supervisión y monitoreo (Oakill, 2003 Cain et al., 2004, Nation et al, 2004 ). Los datos de nuestro estudio aportan que aún cuando la decodificación se inscriba en un contexto de significado a nivel de frase, no es suficiente para hacer la diferencia entre la buena o mala comprensión.

De todos modos nuestros datos también demuestran una relación entre los malos comprendedores y el procesamiento léxico, sólo cuando los aspectos del procesamiento léxicosemántico se agravan (Perfetti, 2008 Nation, Snowling, 1998a; 1999; Nation et al., 2001). Es por ello que discutiremos los resultados de acuerdo a los distintos cuartiles de desempeño.

Los resultados indican que en el nivel de sujetos identificados como MD, no superan la media en comprensión lectora un 67,9\% (Cuarto), un $71,9 \%$ (Quinto) y un $79 \%$ (Sexto).

Parece bastante razonable argumentar que identificamos un rango de rendimiento donde los procesos de decodificación imponen un claro límite a los procesos de comprensión.

Si pasamos al siguiente cuartil en la prueba TECLE, es decir los BD (Percentil 25-50) en- 
contramos que un $62 \%\left(4^{\circ}\right)$, un $61,6 \%\left(5^{\circ}\right)$ y un $33,3 \%\left(6^{\circ}\right)$ son los sujetos que no superan el rendimiento promedio en comprensión. Se observa un dato interesante, pareciera que el límite que la decodificación impone a la comprensión y se sostiene en $4^{\circ}$ y $5^{\circ}$, es superado en el último año de la escolaridad. Es probable que estos alumnos, se hayan beneficiado de las prácticas pedagógicas orientadas al desarrollar estrategias de estudio que en el diseño curricular uruguayo, claramente se intensifican en la última etapa escolar. A su vez, es razonable inferir que hayan desarrollado estrategias alternativas a su debilidad en decodificación, pudiendo acceder a la comprensión con otras vías: inferir significados por el contexto, identificar la estructura textual, operar con señales retóricas, actualizar su competencia lingüística (Cain, 2006, 2007; Sánchez, 2002; Nation, 2007). De todos modos, nuestro estudio presenta limitaciones en cuanto a que si bien demuestra que los alumnos de $6^{\circ}$ terminan por lograr un buen desempeño en comprensión más allá de sus dificultades en decodificación, no nos indica nada acerca de la probable deficitaria relación costo-beneficio que requiera.

En los siguientes cuartiles de decodificación se observa un comportamiento interesante en los tres grados escolares. Con un nivel adecuado de decodificación, todo parece posible en cuánto a los resultados en comprensión. Nuestros datos son coherentes con los postulados clásicos que sostienen que un adecuado nivel de decodificación se muestra necesario, pero no suficiente. Parafraseando a Bertelson (1986) aunque en sentido contrario, estos resultados sugieren que una vez que el lector ha logrado un buen nivel de identificación de palabras, es probable que aún quede todo por hacer para ser un buen comprendedor.

Otro dato interesante de nuestro estudio surge al analizar la correlación entre las diferentes medidas de comprensión (Comprensión y Comprensión 1), que también es moderada. Esta evidencia confirma que ambas tareas ponen en juego habilidades distintas de comprensión, aspecto que estuvo contemplado desde el diseño de la prueba, pero sobre todo, que la mejora de uno de los procesos parece ser independiente del otro, es decir, puede haber disociaciones entre estas diferentes habilidades (Perrig y Kintsch, 1985; Colte, Goldman y Saul, 1998). Es así que los participantes de nuestro estudio pueden lograr una comprensión a nivel del texto base, es decir, comprender la relación entre dos frases tanto contiguas como alejadas, parafrasear con éxito la información contenida en uno o dos párrafos. Sin embargo esto no supone que alcancen tareas que involucren el modelo de situación, en tareas que exigen la aplicación de las macrorreglas de supresión, integración y generalización, como lo es explicar el tema en tres o cuatro frases como máximo y discernir el título adecuado entre cuatro opciones.

Otro de nuestros objetivos era analizar la evolución de la correlación entre decodificación y comprensión en el transcurrir de la escolaridad. Confirmamos nuestra expectativa inicial respecto a que la correlación decrece conforme avanza la escolaridad, pero parece evidente que para identificar una relación más interdependiente entre decodificación y comprensión, deberíamos ir a etapas más tempranas de la escolaridad, tal como se ha observado en otros estudios (Storch y Whitehurst, 2002) o a niveles socio-culturales menos favorables.

Implicaciones educativas se desprenden de nuestro estudio al ratificar la necesidad de intervenir en los procesos de bajo nivel en los primeros años de escolaridad, ya que imponen un claro límite a la comprensión. Por otra parte, la disociación observada una vez que los lectores superan el primer cuartil de desempeño en decodificación, evidencia la necesidad de diseñar intervenciones precisas para mejorar la comprensión oral y escrita, independientes del logro en decodificación. Estudios evidencian que los trastornos del lenguaje oral que no presentan el perfil clásico de trastornos en la fonología y la decodificación, suelen pasar inadvertidos por el sistema escolar en las etapas tempranas, identificándose de forma tardía en los últimos años de escolaridad, por su mal desempeño en comprensión lectora (Kelso et al. 2007). En lo que respecta a los diseños de intervención en comprensión de textos, nuestros resultados evidencian la necesidad de abordar de forma independiente los procesos relacionados con la comprensión del texto-base y aquellos relacionados con el modelo de situación, ya que pudimos observar que realizar inferencias locales y adyacentes entre distintos fragmentos del texto no aseguraba la construcción del modelo de situación. 


\section{REFERENCIAS}

Bertelson, P. (1986). The onset of literacy: liminal remarks. Cognition and Instruction, 24, 1-30, en Alegría, J. (2006). Por un enfoque psicolinguistico del aprendizaje de la lectoescritura: 20 años después. Infancia y Aprendizaje, 29(1), 93-111.

Cain, K., Oakhill, J., \& Lemmon, k. (2004). Individual differences in the inference of word meanings from context: the influence of reading comprehension, vocabulary knowledge, and memory capacty. Journal of Educational Psychology, 96(4), 671-681.

Cain, K., \& Oakhill, J. (2006). Profiles of children wit specific reading comprehension difficulties. British Journal of Educational Psychology, 46, 683-696.

Cain, K. (2007). Deriving word meanings from context: does explanation facilitate contextual analysis? Journal of Research in Reading, 30(4), 347-359.

Cornoldi, C., Benni, R. d., \& Pazzaglia, F. (1996). Profiles of reading comprehension difficulties: an analysis of single cases. In C. e. Cornoldi \& J. Oakhill (Eds.), Reading Comprehension Difficulties: Processes and Intervention (pp. 113-136). New Jersey: Lawrence Erlbaum.

Cote, N., Goldman, R., \& Saul, E. (1998). Students making sense of informational text: Relations between processing and representation. Discourse Processes, 25 (1), 1-53.

Cuadro, A., \& Carballo, M. (2007). La velocidad de nominación en el déficit lector. Paper presentado en el X Congreso Latinoamericano para el Desarrollo de la Lectura y Escritura II Congreso Internacional de la Sociedad de Dislexia del Uruguay., Montevideo

Cuadro, A., Costa, D., Trías, D., \& Ponce de León, P. (2009). Evaluación del nivel lector. Manual Técnico del test de Eficacia Lectora de J. Marín y M. Carrillo. Montevideo: Prensa Médica Latinoamericana.

Cuetos, F.; Rodríguez, B. \& Ruano E. (1998). PROLEC. Evaluación de los procesos lectores. Madrid: TEA.

Georgiou, G. K., Das, J. P., \& Hayward, D. (2009). Revisiting the "Simple View of Reading" in a Group of Children with Poor Reading Comprehension. Journal of Learning Disabilities, 42(1), 76-84.

Gough, P., \& Tunmer, W. (1986). Decoding, reading and reading disability. Remedial and Special Education, 7, 6-10.

Gough, P., \& Tunmer, W. (1996). Some observations on a simple view of reading. In C. Cornoldi \& J. Oakhill (Eds.), Reading Comprehension Difficulties: Processes and Intervention (pp. 1-13). New Jersey: Lawrence Erlbaum.

Johnston, A. M., Barnes, M., \& Desrochers, A. (2008). Reading Comprehension: developmental processes, individual differences and interventions. Canadian Psycology, 49(2), 125-132.

Kelso, K., Fletcher, J., \& Lee, P. (2007). Reading comprehension in children with specific language impairment: an examination of two subgroups. International Journal of Language and Communication Disorders, 42(1), 39-57.

Kintsch, W., \& Rawson, K. (2007). Comprehension. En The Science of Reading. A handbook Blackwell Publishing.

Nation, K. (2007). Childrens Reading Comprehension Difficulties. En The Science of Reading. A handbook: Blackwell Publishing.

Muter, V., Hulme, C., Snowling, M. (2004). Phonemes, Rimes, Vocabulary, and Grammatical Skills as Foundations of Early Reading Development: Evidence From a Longitudinal Study. Developmental Psychology, 40(5), 665-681.

Mouzaki, A., Protopapas, A., \& Spantidakis, I. (2006). The role of oral language developmental in reading comprehension:evidence from greek elementary school students. Paper presented at the Proccedings of the 14 World Congress on LD.

Oakhill, J., Cain, K., \& Bryant, P. (2003). The dissociation of word reading and text comprehension: evidence from component skills. Language and Cognitive Processes, 14(4), 443-468.

Pazzaglia, F., Cornoldi, C., \& Tressoldi, P. (1993). Learning to read: Evidence on the distinction betwen decoding and comprehension skills. European Journal of Psychology of Education, VIII(3), 247-258.

Perfetti, C. (1985). Reading Ability. New York: Oxford University Press.

Perffeti, C. (2007). The Acquisition of reading comprehension skills. In The Science of Reading. A handbook Blackwell Publishing.

Perfetti, C., Yang, C.-L., \& Schmalhofer, F. (2008). Comprehension skill and word-to-text integration processes. Appl. Cognitive Psychology, 22, 303-318.

Perrig, W., \& Kintsch, W. (1985). Propositional and situational representations of text, Journal of Memory and Language, 24(5), 503-518.

Ransby, M., \& Swanson, H. L. (2003). Reading comprehension skills of young adults wit childhood diagnoses of dyslexia. Journal of Learning Disabilities, 36(6), 538-555.

Sánchez, E., Gonzalez, A., \& García, R. (2002). Competencia Retórica. Una propuesta para interpretar dificultades de comprensión. Psicothema, 14(1), 77-85.

Sánchez, E., \& García-Rodicio, H. (2006). Re-lectura del estudio PISA: qué y cómo se evalúa e interpreta el rendimiento de los alumnos en la lectura. Revista de la Educación, extraordinario, 195-226.

Storch, A., \& Whitehurst, G. (2002). Oral language and code-related precursors to reading: Evidence from a longitudinal structural model. Developmental Psychology, 38, 934-947.

Stothard, S., \& Hulme, C. (1992). A comparison og phonological skills in children with reading comprehension difficulties and children with decoding difficulties. Journal of Child Psychology and Psychiatry, 36(3), 399-408. 